\title{
Transmission of vibration through gloves: effects of material thickness
}

\begin{abstract}
It might be assumed that increasing the thickness of a glove would reduce the vibration transmitted to the hand. Three material samples from an anti-vibration glove were stacked to produce three thicknesses: 6.4, 12.8 and $19.2 \mathrm{~mm}$. The dynamic stiffnesses of all three thicknesses, the apparent mass at the palm and the finger and the transmission of vibration to the palm and finger were measured. At frequencies from 20 to $350 \mathrm{~Hz}$, the material reduced vibration at the palm but increased vibration at the finger. Increased thickness reduced vibration at the palm but increased vibration at the finger. The measured transmissibilities could be predicted from the material dynamic stiffness and the apparent mass of the palm and finger. Reducing the dynamic stiffness of glove material may increase or decrease the transmission of vibration, depending on the material, the frequency of vibration and the location of measurement (palm or finger). Practitioner Summary: Transmission of vibration through gloves depends on the dynamic response of the hand and the dynamic stiffness of glove material, which depends on material thickness. Measuring the transmission of vibration through gloves to the palm of the hand gives a misleading indication of the transmission of vibration to the fingers.
\end{abstract}

Keyword: Anti-vibration gloves; Biodynamics; Fingers; Hands; Impedance; Transmissibility 